\title{
RAPID OPTICAL FOLLOW-UP OBSERVATIONS OF GAMMA-RAY BURSTS
}

\author{
MICHEL BOËR \\ Observatoire de Haute-Provence (CNRS) \\ 04870, Saint Michel l'Observatoire, France \\ Michel.Boer@oamp.fr
}

\begin{abstract}
The prompt emission of gamma-ray burst sources is still the main means of detection, and a privilegied access to the souce dynamics. It is detected from radio to $\mathrm{GeV}$ energies, and its study is crucial for the overall understanding of the phenomenom. We present here a panorama of the rapid optical observations, and what can be infered from the data. We will discuss also the new instruments which are planned for the observation of the prompt and early afterglow at optical and infrared wavelengths, with spectral capabilities.
\end{abstract}

Keywords: Gamma-ray bursts.

\section{Introduction}

Gamma-ray bursts (hereafter GRBs) are high energy events composed of a prompt phase lasting from less than a tenth of a second to minutes; It is followed by a gradually decaying phase, the afterglow ${ }^{1}$, with a X-ray emission decaying as $\mathrm{t}^{-1,-2}$ in first approximation (Fig. 1).

GRBs were discovered in the late sixties $^{2}$; their detection relies still mostly on their high energy prompt emission. The rate of detection (corrected from instrumental biases) is roughly 2 events per day for a threshold fluence of $10^{-8} \mathrm{erg} . \mathrm{s}^{-1} . \mathrm{cm}^{-2}$ over the entire sky. This number transforms in a rate of $80 / y$ for the SWIFT spacecraft and 200/y for the Fermi GBM experiment, or to a density of $2 \mathrm{Gpc}^{-3} \mathrm{y}^{-1}$.

Though GRBs are in essence panchromatic events, detected up to $30 \mathrm{GeV}$, their spectrum peaks between $100 \mathrm{keV}$ to $1 \mathrm{MeV}$ (Fig. $2^{3}$ ), making them a prototype of high energy sources. They are also characterized by a very rapid variability, down to the millisecond scale.

When it was realized that GRBs were located at cosmological distances ${ }^{4}$ physicists had to explain the origin of the tremendous energy apparently released, on the order of $10^{52-53} \mathrm{erg}$. Combined with rapid, submillisecond variations in the profile, this leads to the pair opacity problem for photon-photon interactions. In addition, one of the difficulties is to explain the large dispersion of the luminosities implied by the huge distance range, from redshift of 0.001 to 8.3 . 


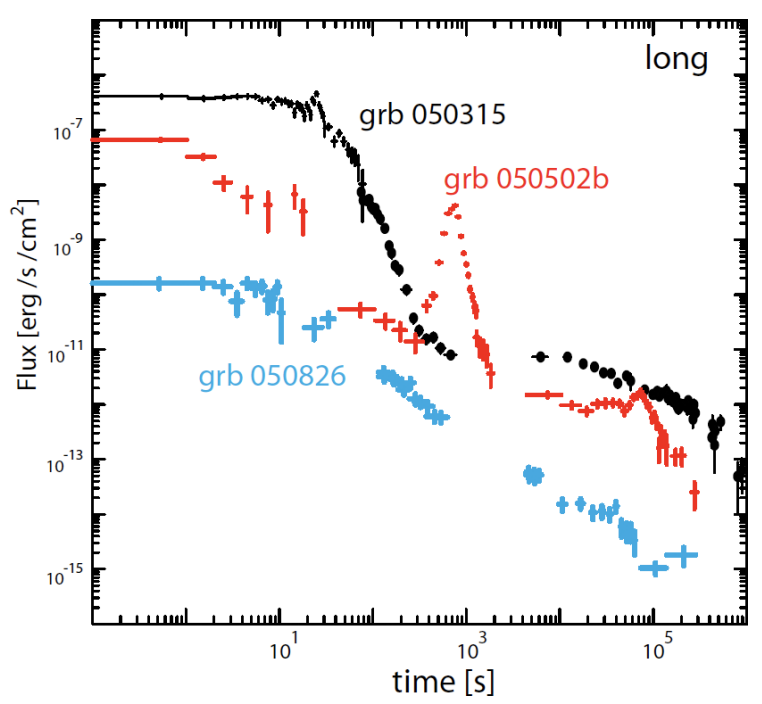

Fig. 1. Three GRB light-curves in the hard X-ray range obtained by combining data from Swift/BAT (15-150 keV) and Swift/XRT (0.2-10 keV). GRB 050315 shows a clear separation between the prompt emission, which ends with an exponential decay around $\mathrm{T} \sim 100 \mathrm{sec}$, and a plateau phase preceding the decaying afterglow. GRB 050826 shows a smooth transition between the prompt emission and the afterglow at $\mathrm{T} \sim 10 \mathrm{sec}$. GRB 050502B shows a very bright X-ray flare at $\mathrm{T} \sim 700 \mathrm{sec}$ superimposed on the canonical decay of the afterglow - From Gehrels et al. (2009).

This apparent paradox can be solved if we suppose that GRBs are emitting in jets pointing towards our direction, with Lorentz factor of several hundreds, however at the expense of a large increase of source numbers.

A popular model for GRBs is the so-called fireball model where the prompt emission is produced by internal shocks within the jet, and the afterglow is due to its interaction with the ambiant medium. How the energy is dissipated is still debated: magnetic energy reconection or relativistic shocks are often invoked leading to synchrotron radiation (see e.g. Ref. 5 for a review). However, the detection of gamma-ray emission implies that the firebal has become transparent, and that most of its energy has been radiated at the level of the photosphere.

In the following we present the information which can be gathered from rapid optical observations of the prompt event and of the early afterglow. For a more comprehensive review of the prompt event at all wavelengths the reader is refered to Atteia and Boër $(2011)^{6}$.

\section{The prompt emission and early afterglow in optical}

\subsection{What is the prompt emission?}

When we use the term of "prompt emission" we usually refer to the bright flash of gamma-rays which precedes the afterglow. The first problem is that when looking at 


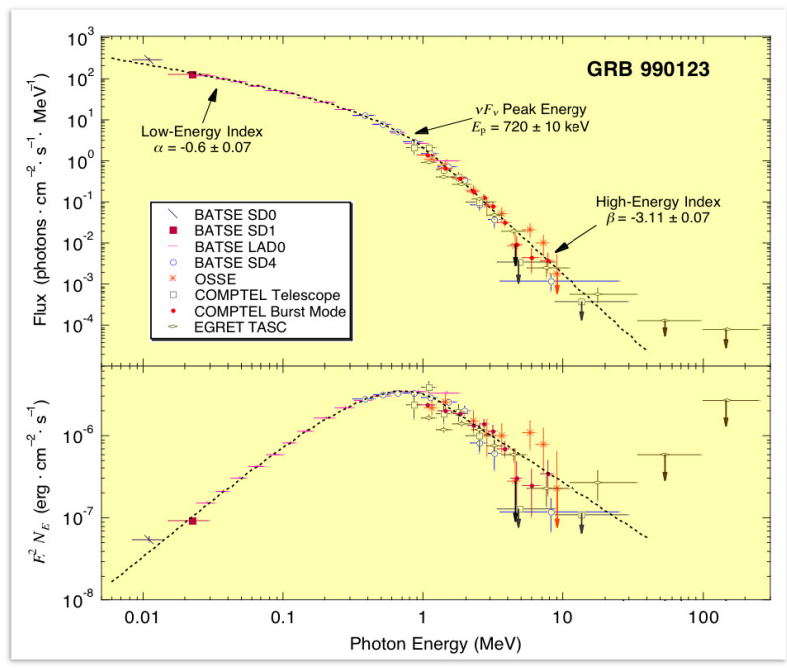

Fig. 2. High energy spectrum of GRB 990123 From Briggs et al. (1999).

data at several wavelengths, mostly X-rays, the afterglow starts usually as a second component of the prompt emission (see e.g. Fig. 1). Moreover the light curve may be quite complex, with quiscent periods for 10 or 100s in some GRBs. Finally, the presence of precusor emission in a variety of cases challenges this simple view of the prompt emission.

Another possibility is to define the prompt phase as connected to the activity inside the jet, whatever it is, e.g. internal shocks. However this means defining a physical, observed phenomenom using the (eventually controversial) theoretical model which is supposed to explain it.

In the following we will use the simple, though not satisfactory, definition of the prompt emission as the flash of gamma-rays recorded by high energy instruments.

\subsection{Some remarks}

Thanks to the rapid dissemination of GRB localizations from BATSE with BACODINE (now called the GCN, GRB Coordinate Network ${ }^{7}$ ) it became possible in the late nineties to observe GRB positions within seconds after the high energy emission triggers the experiment. However, the intrinsic limitation of BATSE in accuracy (on the order of 10 deg.) limited the efficiency of the search. Nowadays the GCN distributes advanced notice to various users, from direct, "socket", connections for automated telescopes to e-mails and reports for the teams observing at large facilities in optical, radio or even Cerenkov detectors.

The first event detected at optical wavelengths was GRB $990123^{8}$ which is still one of the best studied cases. Obviously, as noted at all wavelengths, the observed 
brigthness is dominated by the intinsic luminosity rather than by the distance: GRB 050904b reached magnitude $\mathrm{R}=14^{9}$ for a redshift of $\mathrm{z}=6.2$.

Observing the prompt emission, even with telescopes as rapid as TAROT $^{10}$ (Rapid Action Telescope for Transients Objects, Télescope à Action Rapide pour les Objets Transitoires) is rare because of the time between the actual trigger onboard the satellite and the reception of the alert, with Swift more than 20s, the availability of the telescope (day-night cycle, weather...), and the position of the source (above local horizon). About $10 \%$ of the alerts can be effectively followed.

On TAROT, on the 60 alerts which where processed since 2002, only 14 could be observed during the prompt phase, leading to 5 positive detections.

The simultaneous observation of the prompt phase at gamma-ray, X-ray and optical wavelengths is important since it gives access to the source dynamics. In the "classical" fireball model, the start of the reverse shock can be observed at optical wavelengths.

The transition between the prompt and the afterglow, difficult to observe in gamma-rays, but often observed at optical wavelength, is the sign of the start of the forward shock. This enables to make a tomography of the medium surrounding the progenitor, leading to some indication on its nature and eventually its mass.

Finally, the common observation of the prompt gamma-ray burst together with a burst of neutrino on ICECUBE or km3, or with a LIGO/VIRGO signal, may not

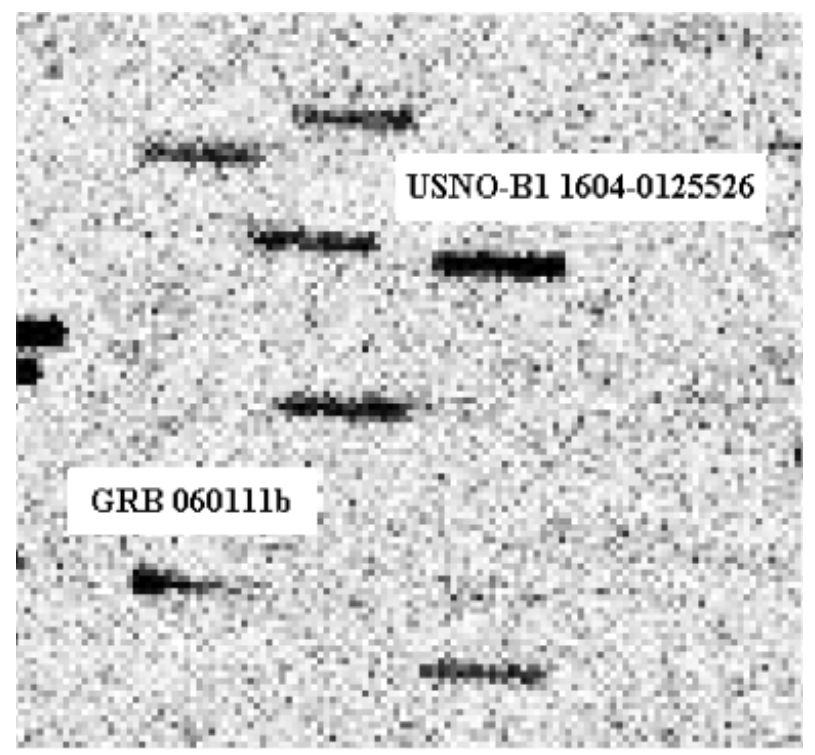

Fig. 3. First TAROT image of GRB 060111 illutrating the special trailing mode which allows for the measurement of continuous light curves during the first 30s. The fluctuation of GRB 060111 are clearly visible compared to the other, standard stars. USNO-B1 1604-0125526 has been taken as a reference star. 
only be the definitive proof of the detection, but it enables much more sensitive searches with these instruments.

\subsection{GRB 060111: the first continuous light curve of the prompt optical event}

One of the problem facing the "rapid" optical observer is the deadtime associated with the readout of the CCD cameras. It is of course possible to use other devices such as EMCCDs, but in addition to their high price, these cameras are still limited in size and difficult to operate in the environment of a rapid GRB telescope.

On TAROT we are using a work-around: the first image of the series is trailed, such that any object has an 18 pixel trail in the E-W direction of the CCD, i.e. each pixel represents a time bin of $3.3 \mathrm{~s}$. The price to pay is a reduced sensitivity (about $1 \mathrm{mag}$ ), but to date TAROT has proven to be the only optical telescope able to produce GRB optical light curves without dead time.

The power, and the interest of the method is illustrated for GRB $060111^{11}$ in Fig. 3. One can easily see the GRB source against the various stars. From the image we were able to derive the light curve (Fig. 4), showing the continuous decay at optical wavelengths, even during the first part of the burst.

We could study in detail this burst, located at a redshift of 2, with an Av of 2.7 magnitudes, showing evidences for a reverse shock with probably a Lorentz factor of 200 to $300^{12}$.

\subsection{GRB 081126: a shift between gamma-rays and optical}

Another case illustrates the usefulness of having continuous light curves: GRB $081126^{13}$.

In this case, thanks to an early alerts, we were able to start observations only $20 \mathrm{~s}$ after the Swift trigger. Contrary to the previous case the optical light curve follows

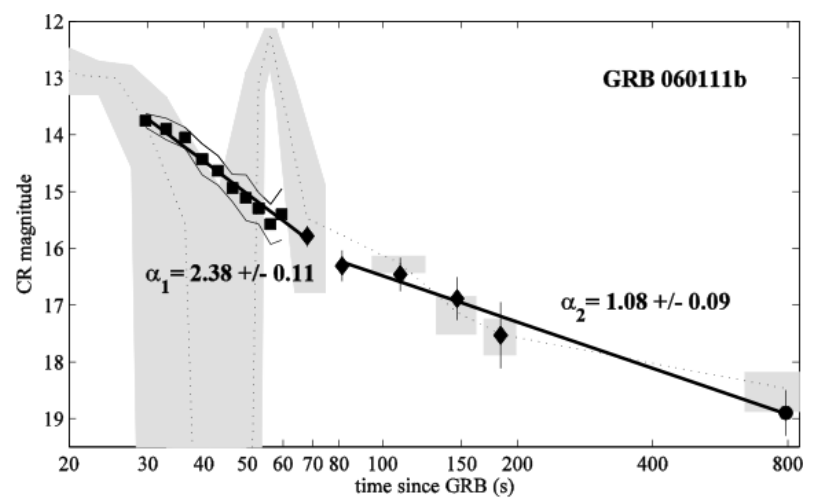

Fig. 4. Light curve of GRB 060111 from TAROT data. The gray curve represents the gamma-rays as recorded by the Swift BAT detector. 


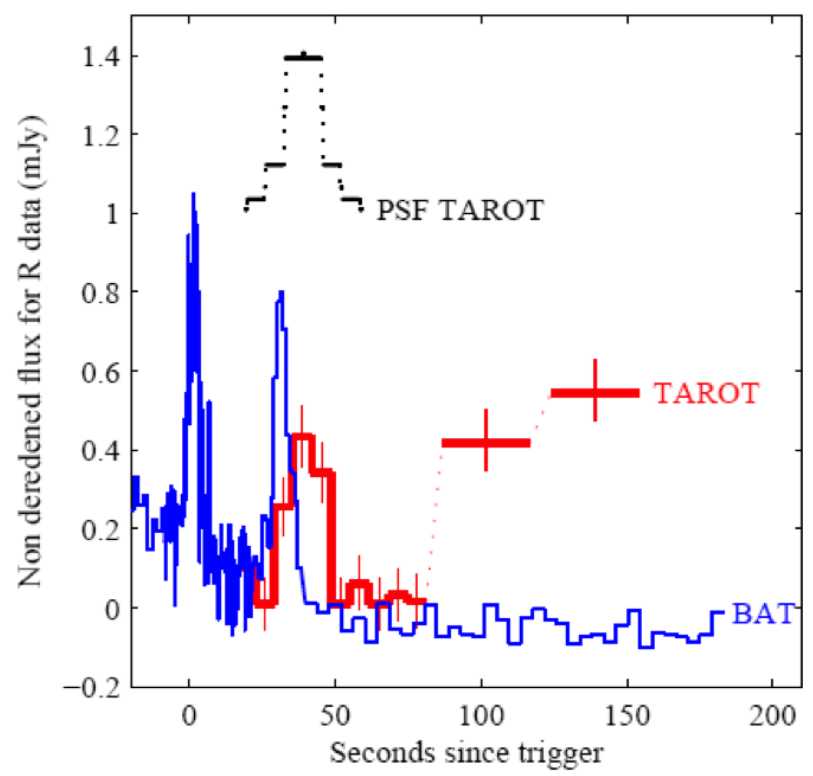

Fig. 5. Light curve of GRB 081126 from TAROT data. The blue curve represents the gamma-rays as recorded by the Swift BAT detector, while the TAROT light curve is plotted in red.

the gamma-rays (Fig. 5), but with a time lag of $8.4 \mathrm{~s} \pm 3.9 \mathrm{~s}$. This strong correlation, together with the time lag, remains difficult to explain within the canonical fireball model.

\subsection{The peculiar light curve of GRB 090102}

An even stranger case come from GRB $090102^{14}$. The optical light curve is uniformly smooth, has a "steep" to "flat" trend, with a break at 1000s (see Fig. 6). There is no strong absorption in the visible spectrum. Contrary to the apparences, the X-ray behaviour is different, with a steeper index, and evidences for absorption in the spectrum.

We try to apply the closure relations to this burst, and we failed to reconciliate the data of this very peculiar burst to the standard model.

\subsection{Can we derive common trends in optical light curves?}

Since the 2002 TAROT has observed 61 burst sources. Among them, we were able to point 13 position while the prompt phase was still active. 16 GRB sources where positively detected, and 6 during the prompt phase. Fig. 7 shows all the TAROT observations in a synthetic way, with the light curve plotted whenever possible, otherwise an upper limit at the time of the first TAROT observation.

As it has been done for SWIFT ${ }^{15}$ it is possible using TAROT data to derive a some common trends in GRB optical counterpart light curves ${ }^{16}$. This is displayed in 


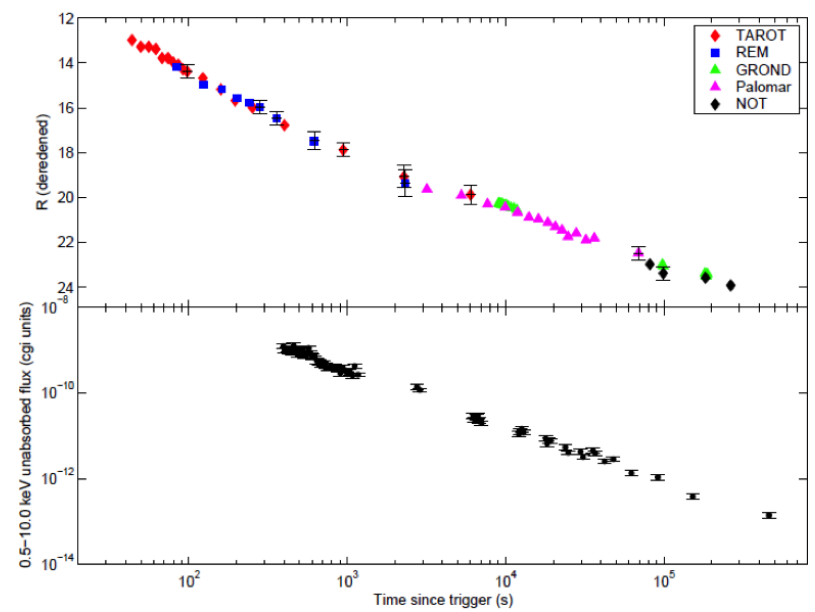

Fig. 6. Light curve of GRB 090102 from TAROT and other telescopes data (see references in Gendre et al., 2010), against the Swift X-ray light curve.

Fig. 8. One should note that despite the apparent analogies, the behaviour is usually different in optical and X-rays. Models such as the refreshed shock have difficulties in interpreting these differences, since they require to have similar profiles at all wavelengths.

\section{The future of rapid observations}

As GRB are panchromatic sources, they need to be observed at all wavelengths, from radio to $\mathrm{GeV}$ and $\mathrm{TeV}$ gamma-rays. At radio wavelengths, $\mathrm{SKA}^{17}$ and $\mathrm{LOFAR}^{18}$ will open a new window on searches for radio transients, providing invaluable data on the lorentz factor of the fireball, the magnetic fields, etc. At the other end of the electromagnetic spectrum the LAT experiment onboard the Fermi spacecraft is already providing invaluable, and puzzling data ${ }^{19}$, confirming the early findings of CGRO-EGRET ${ }^{20}$ that GRB where emitting GeV photon both at the time of the main keV-MeV prompt event, and long (minutes to hours) after.

The observation of the optical part of the GRB spectrum remains of paramount importance since this is still the main way to get a redshift measurement, a very precise localization, absorptions, spectral evolution, etc.

We can see several ways to increase the efficiency of the rapid optical observations:

- A better distribution of telescopes with a comprehensive and consistent set of telescope, around the Earth, in order to increase the detection probability and the contunuous coverage of the light curve: this is the purpose of the CADOR ${ }^{21}$ (Coordination And Data Analysis for Robotic Observatories - Coordination et Analyse des Données pour les Observatoires Robotiques) network of telescopes, which links the two TAROT telescopes in France and Chile, the Zadko 1m near 


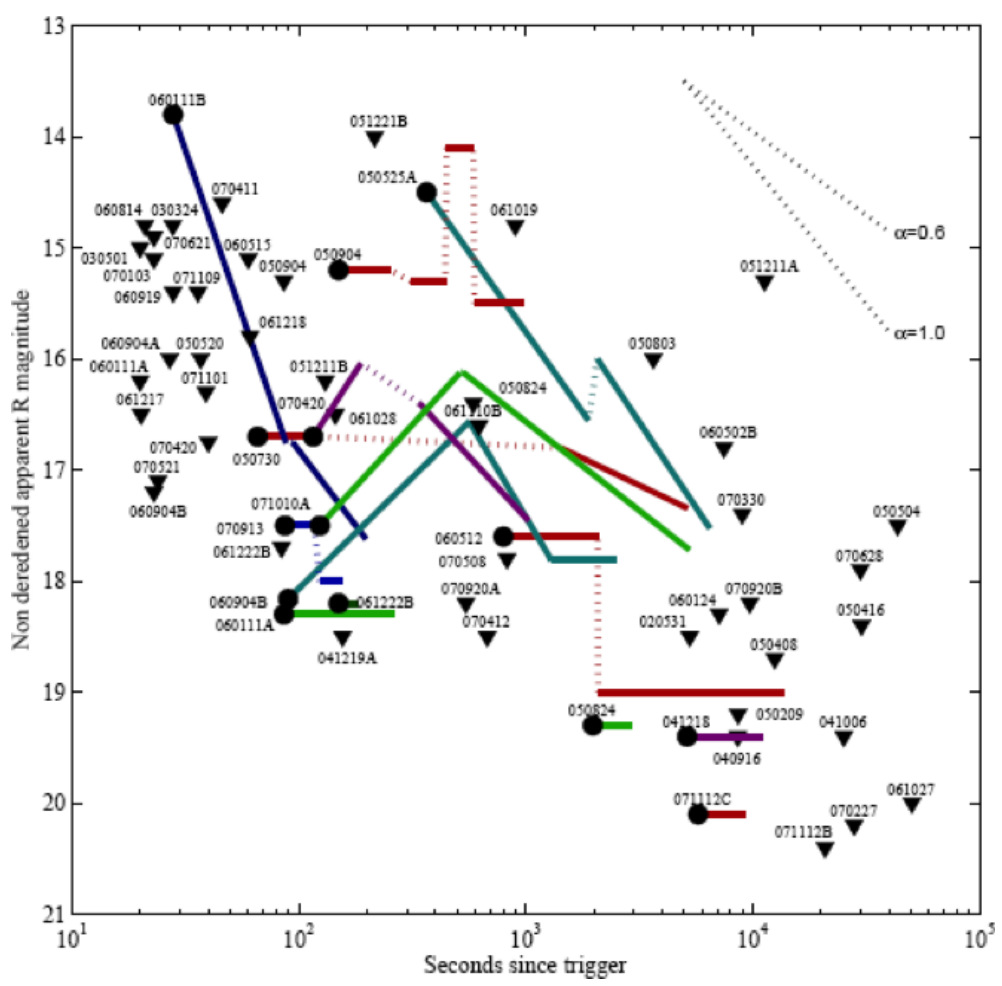

Fig. 7. All observations of GRB sources from TAROT. Whenever possible we plotted the actual light curves against the time after the Swift trigger, otherwise the upper limit at the time of the first measurement from TAROT

Perth, Western Australia, and several other instruments in France. Several instruments will be added in the near future, increasing the coverage in longitude and latitude. CADOR provides a means to centralize the data over a distributed network of telescopes and to optimize the GRB source coverage.

- Increase the sensitivity of the telescopes, i.e. larger apertures: ideally, about two third of the sources should be positively detected within one minute after the trigger. This is the purpose of the existing $1 \mathrm{~m}$ Zadko telescope $^{22}$ in Gingin, near Perth, Western Australia, which was already used in conjunction with the TAROT-Chile telescope through the CADOR network. We plan also to implement a new $1 \mathrm{~m}$ telescope as a follow-up of the forthcoming SVOM Chinese-French GRB mission ${ }^{23}$.

- Access to the infrared domain is of paramount importance, because of the reduced absorption and of the importance of the spectral information and processes, and also to be able to detect routinely GRBs which are at redshifts in the range 5-15. This is the main purpose of the planned 1m GFT telescope (Ground Followup Telescope), within the French-Chinese SVOM mission; the GFT will have an infrared camera. 


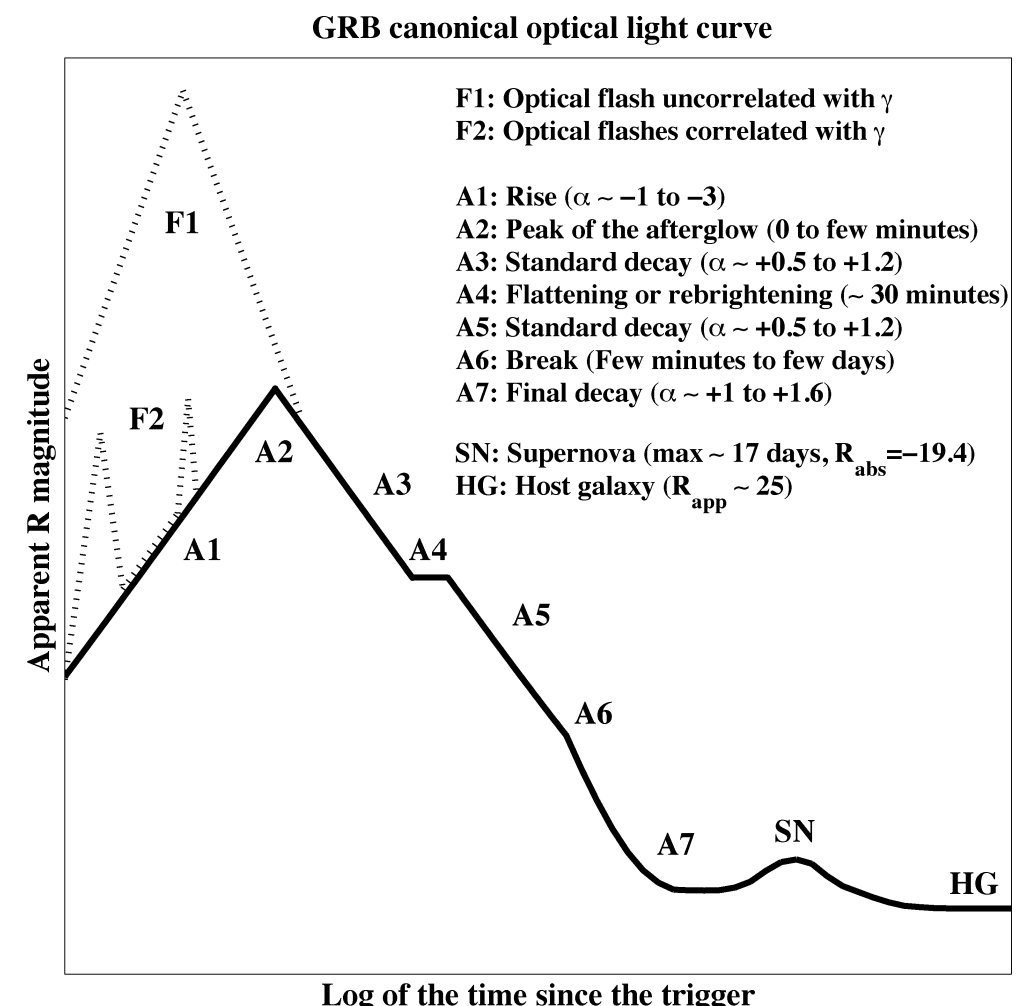

Fig. 8. The "canonical" TAROT light curve for GRBs.

- Simultaneous spectral capabilities (say resolution about 100), i.e. derive a spectrum from the blue to the infrared, simultaneously. The above mentionned GFT will be equiped, at its optical focus, with a resolution 100 spectrograph. This will be the only instrument able to give time resolved spectra within seconds after the start of the burst.

\section{Conclusion}

We have seen that optical observations starting at the time of the prompt gammaray event are of importance for the comprehension of the physics of the GRB. They give access to the source dynamics and the reverse shocks.

The observations obtained by small telescopes like TAROT display both a common trend and some diversity. The optical prompt and early afterglow do not follow usually the X-ray afterglow behaviour, leading to problems when one wants to reconcilate the observations with the current theories. This is the case also for high energy observations from Fermi-LAT.

For the future, it is important to enhance the Earth coverage with rapid telescope, to explore the prompt in infrared and to get time resolved spectra of the 
prompt, as well as of the early afterglow part of the GRB. This is the purpose of the Ground Follow-up Telescope project which should acquire data within seconds after the start of the burst.

\section{Acknowledgments}

The TAROT project has been funded by CNRS-INSU and built with the support of the CNRS-INSU Technical Division. The author acknowledges also the donation of the $1 \mathrm{~m}$ Zadko Telescope at UWA by Dr. Jim Zadko, giving an invaluable help for the study of GRBs.

\section{References}

1. Gehrels, N., Ramirez-Ruiz, E., and Fox, D.B.: 2009, Annual Review of Astronomy and Astrophysics 47, 567.

2. Klebesadel, R.W., Strong, I.B., and Olson, R.A.: 1973, Astrophys. J. 182, L85.

3. Briggs, M.S., Band, D.L., Kippen, R.M., et al.: 1999, Astrophys. J., 524, 82

4. Costa, E., Frontera, F., Heise, J., Feroci, M., in't Zand, J., et al.: 1997, Nature 387, 783.

5. Meszaros, P.: 2006, Rev. Prog. Phys., 69, 2259

6. Atteia, J.M., and Boer, M.: 2011, CR Acad. Sci., in press

7. Barthelmy, S.D., et al.: 1994 in "Proceeding of the 2nd Huntsville Workshop", eds. G.Fishman, J.Brainerd, K.Hurley, AIP Conference proceedings 307, p. 643.

8. Akerlof, C., Balsano, R., Barthelmy, S., et al.: 1999, Nature 398, 400.

9. Boer, M.; Atteia, J. L.; Damerdji, Y., et al.: 2006, Astrophys. J. 638, L71

10. Boer, M., Atteia, J.-L., Bringer, M. et al.: $2000 A \mathscr{E}$ A, 378, 76

11. Klotz, A.; Gendre, B.; Stratta, G., et al.: 2006 A\& A, 451, 39

12. Stratta, S., Pozanenko, A.; Atteia, J.-L. et al.: 2009 A 6 A, 503, 783

13. Klotz, A.; Gendre, B.; Atteia, J.L., et al.: 2009 ApJ, 697, L18

14. Gendre, B., Klotz, A., Palazzi, E. et al.: 2010 MNRAS, 405, 2372

15. Nousek, J.A., Kouveliotou, C., Grupe, D., et al.: 2006, ApJ, 642, 389.

16. Klotz, A., Boer, M., Atteia, J.-L., Gendre, B.: 2009, Astron. J., 137, 2100.

17. Fender, R., Wijers, R., Stappers, B., 2007, in "Bursts, Pulses and Flickering: wide-field monitoring of the dynamic radio sky", Tsioumis, Laszos and Fender eds., Proceedings of Science, SISSA, available online at http://pos.sissa.it/cgibin/reader/conf.cgi?confid $=56$.

18. Weiler K.W.,Van Dyk D.S., Sramek, R.A., and Panagia, N.: 2006, New Astron. Rev., 48, 1377.

19. Band, D.L., Axelson, M., Baldini, L.: 2009, ApJ, 701, 1673

20. Hurley, K., Dingus, B.LL., Mukherjee, R., et al.: 1994, Nature, 372, 652

21. Bourrez-Laas M., Vachier, F., Klotz, A. et al.: 2008, in "Advanced Software and Control for Astronomy II", Eds. Bridger, A.; Radziwill, N.P., Proceedings of the SPIE 7019,40

22. Coward, D., Todd, M., Waalsta, T.: 2010, PASA, 27, 331

23. Schanne, S.; Atteia, J.-L., Barret, D.: 2008, Nucl. Inst. Meth., A567, 327 